\title{
Gerontology
}

Gerontology 2011;57:559-564

DOI: $\underline{10.1159 / 000323481}$
Received: June 29, 2010

Accepted: December 1, 2010

Published online: February 11, 2011

\section{Neurogenesis, Cellular Plasticity and Cognition: The Impact of Stem Cells in the Adult and Aging Brain - A Mini-Review}

\author{
Sebastien Couillard-Despres ${ }^{a}$ Bernhard Iglseder ${ }^{b} \quad$ Ludwig Aigner $^{a}$ \\ ${ }^{a}$ Institute of Molecular Regenerative Medicine, and ${ }^{b}$ Department of Geriatrics, Christian Doppler Clinic, \\ Paracelsus Medical University Salzburg, Salzburg, Austria
}

\section{Key Words}

Aging • Neural stem cells • Neurogenesis • Hippocampus •

Cognition $\cdot$ Learning

\begin{abstract}
The hippocampus is a structure equipped with a high degree of flexibility and adaptation. In contrast to most structures of the adult central nervous system, the hippocampus can rely on a form of plasticity known as neurogenesis. The continuous provision of new neurons derived from resident adult neural stem cells appears to facilitate the execution of hippocampal-dependent tasks since reduction or blockage of neurogenesis is associated with cognitive impairments. Importantly, however, although hippocampal neurogenesis is maintained all throughout life, its levels decrease steadily along with aging. Notwithstanding some evidence that in age-matched animals neurogenesis levels and learning performance are tightly associated, these two parameters do not appear to be directly coupled when comparing individuals of various age groups. Additional components, and in particular experience, appear to play a fundamental roles in hippocampal functions. In this review, we speculate on the impact of neurogenesis level modulation on cognitive performances, putting in perspective recent studies made in the aging human population and in rodent models of aging.

Copyright ๑ 2011 S. Karger AG, Basel
\end{abstract}

\section{KARGER}

Fax +4161306 1234

E-Mail karger@karger.ch

www.karger.com
2011 S. Karger AG, Basel

0304-324X/11/0576-0559\$38.00/0

Accessible online at:

www.karger.com/ger

\section{Neurogenesis in the Adult Brain}

Detection of adult neurogenesis in the human central nervous system (CNS) was received with much excitement [1]. It was predicted that in the near future neural stem cells could be the basis for cell replacement and regenerative therapies for the diseased brain or for improvement of cognitive function during aging. However, several issues remain untouched, in particular questions regarding the nature and physiological function of these stem cells. Moreover, their maintenance, relevance and functions in the course of aging still raise questions.

Although the presence of adult neural stem cells has been suggested throughout the mammalian CNS, their identity in vivo remains to be deciphered. There are some major obstacles to their identification, i.e. the lack of univocal markers specific for stem cells and the quiescent state in which stem cells remain most of the time. In rodents and humans, however, neural stem cells are constitutively active in two specific regions of the adult CNS, namely the subgranular lining of the dentate gyrus and the subventricular regions of the lateral ventricles (fig. 1). Under physiological conditions, stem cells proliferate in these two regions to give rise to progenitors, which differentiate predominantly into new neurons [2]. Nevertheless, we and others have demonstrated that these multipotent cells could, in addition to neurons, generate astrocytes and oligodendrocytes as well [3]. 


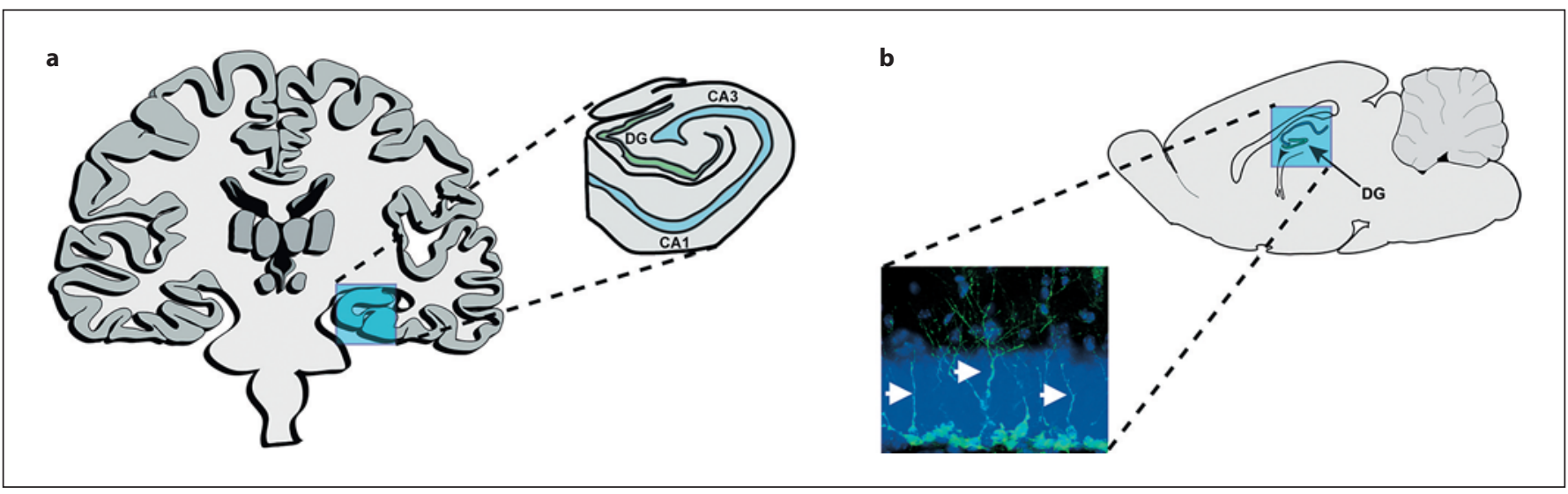

Fig. 1. Localization of the hippocampus in the human brain (a) and the rodent brain (b). Inset in a shows the hippocampal subregions: the dentate gyrus granule cell layer (DG) and the pyramidal cell layer from CA1 to CA3. Inset in b shows immunohistological detection of newly generated neurons (arrows pointing to DCXexpressing cells) in the granule cell layer (nuclei counterstained with DAPI).

Neurogenesis is a process modulated by several molecular and physiological factors. For example, specific molecules such as the growth factor VEGF can promote neurogenesis [4]. Similarly, intensification of voluntary physical activity and exposition to a particularly rich environment have been shown to significantly stimulate neurogenesis $[5,6]$. On the other side, dramatic decline of neurogenesis rates occurs throughout aging or can be induced by intense stress [7-9]. As the function of adult neurogenesis has yet to be deciphered, one can only speculate on the impact of neurogenesis modulations.

It is noteworthy to point out that structures in which new neurons integrate are also active sites of neuronal apoptosis [10]. At the origin of these apoptotic profiles is the fact that only a fraction of newly generated neurons integrate and survive [11]. In addition, resident neurons can also undergo apoptosis and be replaced by the new ones, thereby giving rise to a continuous neuronal turnover in neurogenic regions. Hence, the balance between addition and removal under physiological conditions results in a constant number of neurons within the dentate gyrus [12].

The fact that physiological neurogenesis takes place in the adult hippocampus, probably one of the most investigated regions in the brain, represents a significant advantage for understanding the function and relevance of neuronal precursors. Moreover, it facilitates the development of interventions relying on neural stem cells and neuronal precursors to improve the functional performance. In contrast, in order to integrate neuronal precursors into most other structures of the brain, grafting of exogenous material would be required. Grafting of stem and precursor cells is associated with serious safety issues such as the risk of rejection, tumor formation, epileptic focus formation, etc.

\section{Function of Adult Hippocampal Neurogenesis}

The hippocampus is a crucial structure for cognition and emotions. The prime importance of these functions in humans may explain why most efforts have been deployed in understanding the function of hippocampal neurogenesis. In the hippocampus, the dentate gyrus constitutes a bottleneck for the entry and further processing of information. A prevailing assumption in the field of adult neurogenesis is that the continuous generation of new neurons within the hippocampus offers, in addition to synaptic plasticity, a cellular flexibility to the system. This supplementary adaption capacity could be especially crucial for a structure involved in memory and learning. Indeed, newly generated neurons are particularly excitable and can therefore be more sensitive to incoming input $[7,13]$. Nevertheless, the hypothesis that 'more is better' in respect to neurogenesis has been challenged by recent reports and therefore should not be considered an established fact $[14,15]$.

A definitive demonstration that newly generated neurons are essential for learning is still awaiting. Nevertheless, the bulk of studies support the concept that young neurons contribute and facilitate hippocampal-dependent task learning (e.g. [16-18]). This assistance could be 
mediated, for example, through the lower threshold required for the induction of long-term potentiation in newly generated neurons [19]. We and others have demonstrated that newly generated neurons are integrated very rapidly in the hippocampal network and that they can trigger action potentials upon stimuli that were 10 times weaker than those required to stimulate mature granule cells $[7,13]$. Hence, the presence of numerous immature neurons in the hippocampal network might provide a higher sensitivity to incoming inputs, thereby facilitating information processing.

Various approaches to obliterate the generation of new neurons, such as low-dose irradiations or cytostatic/cytotoxic drugs, have led to cognitive impairments. However, these types of experiments are rather crude in their repercussions and do not exclusively target new neurons [20-22]. Further evidence comes from the modulation of neurogenesis through physiological factors (physical activity, stress, aging) or through transgenic approaches, demonstrating that hippocampal-dependent learning is facilitated by the presence of young neurons [23-26].

Interestingly, a few animal models have been presented in which adult hippocampal neurogenesis appears to be absent or occur at a very low rate. For instance, a mouse bearing a targeted deletion of the cyclin $\mathrm{D} 2$ seemed to lack adult neurogenesis [27, 28]. In this mouse model, the integration of new mature neurons appears to be abolished by the deletion of the cyclin D2 expression; interestingly, however, few DCX-expressing neuronal precursors, representing approximately $10 \%$ of the normal amount, were still being produced. In these neurogenesis-deficient mice, learning and anxiety levels appeared to be virtually indistinguishable from those in their wildtype littermates [27]. One can only speculate about the possibility that the presence of a few remaining neuronal precursors is sufficient to act as facilitators for learning. Nevertheless, the fact that cyclin D2-deficient mice can learn efficiently demonstrates that learning is not dependent on circuitries composed of new neurons.

Two additional models of naturally occurring deficiency in hippocampal neurogenesis are constituted by some species of shrews and bats $[29,30]$. Although cell proliferation or the presence of neuronal precursors cannot be detected in the hippocampi of these species, adult neurogenesis is nevertheless taking place in the subventricular regions, providing the olfactory bulbs with newly generated neurons. Despite the absence of hippocampal neurogenesis, these animals must, for example, integrate and memorize new locations of food supplies, which can be, in the case of some bat species, spread over a very large territory. It is conceivable that the short period of adult neurogenesis which takes place in early adulthood suffices to optimize the hippocampal network for the stereotypic behaviors for the rest of the animal lifespan. Taken together, this evidence indicates that neurogenesis is not a prerequisite for learning; however, a facilitating role of neurogenesis in learning cannot be excluded.

\section{Neurogenesis in the Aged Brain}

Aging is associated with a significant decline of neurogenesis. As a consequence, rates of new neuron generation in aged brains represent only a few percentage points measured in young adults $[8,31,32]$. Notably, however, looking at a cohort of normal aged rats, Bizon et al. [15] and Bizon and Gallagher [32] noticed significant interindividual variation of the learning capacity. Some aged rats (25 months of age) maintained a learning index comparable to that observed in younger animals (7 months of age) even though their neurogenesis rates represented only $15 \%$ of the rates observed in the younger rats [15]. Paradoxically, post hoc analysis revealed that aged 'good learners' from this study exhibited lower rates of hippocampal neurogenesis as compared to the aged 'bad learners'. Moreover, no differences in the total number of hippocampal neurons could be observed between aged good and bad learners [12].

On the other hand, it was recently shown that a transient reduction of neurogenesis rates in young mice by approximately $50 \%$ had significant consequences on learning, although the remaining neurogenesis levels were still several times higher than those present in aged animals [16]. In addition, since the first report made by Kempermann et al. [5], the promoting effect of an enriched environment on neurogenesis and the associated benefits on learning have been described in a plethora of studies and under various paradigms. Similarly, task learning has also been reported to stimulate neurogenesis [17], and, interestingly, the extent of the neurogenesis increase appeared to correlate with the learning demands [18]. Hence, in the face of increased learning pressure, neurogenesis becomes induced according to the need required. Thus, these observations lead to the following question, 'Is adult neurogenesis fulfilling the same function in the young versus the aged brain?'

A way to reconcile the seemingly discrepant observations is to hypothesize that adult neurogenesis keeps fulfilling the same function throughout life, but the requirements of the system change with aging. It is noteworthy 
that although all cellular components of the hippocampus have been set in place shortly after birth, a key element is still missing - 'experience'. An analogy could be made with the immune system, which gets activated and adapted to modify its cellular composition according to the challenges from the environment. Age-associated transformation in hippocampal connectivity has been suggested based on a detailed morphological characterization of the molecular layer [33].

Hence, despite the fact that the overall thickness of the molecular layer remains constant between groups of adult and aged rats, a significant reduction of the medial molecular layer (projections from the medial entorhinal cortex) to the benefit of the inner molecular layer (commissural/associational inputs arising from hilar neurons) can be described. These volumetric changes cannot be attributed to changes in neuronal or glial cell numbers, but rather reflect synaptic reorganization and a permanent rewiring of the hippocampus [33]. It can be speculated that through the experience-guided gradual optimization of the hippocampal information-processing circuitry, the need for cellular plasticity would proportionally decrease. Nevertheless, neurogenesis should remain inducible in cases where individuals are exposed to new challenges. Induction of hippocampal neurogenesis could be the cellular response to the need of integrating new information and adapting to an unknown environment.

Taken together, these observations suggest that cognitive deficits could be the consequence of an imbalance between acquired/integrated experiences and the levels of neurogenesis. Upon exposure to a stimulus, young individuals with poor experience would require high levels of neurogenesis to optimize the circuitry for the new information to be processed. In contrast, in aged individuals, existing circuitries developed and optimized for similar tasks can be reused and may require only minimal adaptation. Therefore, within a cohort of young individuals, those with the highest level of neurogenesis would have the highest capacity to adapt their hippocampal circuitry and would have an advantage in acquisition. Such an observation has indeed been reported following an increase or decrease of neurogenesis levels in young mice $[16,34]$. On the other side, cognitive function in aged animals can still be intact although the remaining levels of neurogenesis are only few percent of those seen in the young ones $[15,31$, 32]. Therefore, although neurogenesis rates appear crucial in learning performance when comparing animals within the same age group (horizontal), additional components are at least as important in the cognitive performance when comparing animals of various age groups (vertical).
Paradoxically, high levels of neurogenesis in aged individuals relative to their age-matched peers may be interpreted in two diametrically opposite ways. On one side, an aged individual may have induced the remaining neurogenic resources more efficiently than his/her peers, resulting in better learning. On the other side, steady high levels of neurogenesis may reflect a life-long failure of the hippocampus to become optimized for incoming inputs and to acquire experience. In this situation, high neurogenesis levels in an aged individual could be associated with a decreased learning performance. Studies supporting these two possibilities have been reported using aged mouse and rat models involved in learning paradigms (e.g. $[15,18,35])$. However, the regulation of neurogenesis during the learning tests has been shown to be complex, involving enhanced or decreased survival of neuronal precursors depending on their maturation stages [18]. The latter, as well as age, sex, strain and species differences, in animals used in published studies complicate comparison and interpretation of the results.

\section{Neurogenesis and Aging in the Human Brain}

Extrapolating observations made in rodent models to the human is always hazardous. Nevertheless, it has been convincingly demonstrated that neurogenesis is also present in the human dentate gyrus [1] and that neurogenesis levels decrease with age. Prevention or slowing down of cognitive decline is of paramount importance for successful aging. Therefore, analysis of functional relevance of neurogenesis in the cognitive performances of the aging brain becomes inevitable. It is noteworthy that stronger challenge of the cognitive resources in young individuals appears to protect them against cognitive decline during aging, and reciprocally lower educational attainment was consistently associated with higher risks for cognitive decline and dementia in numerous studies (e.g. [36]). Several mechanisms may contribute to this observation. For instance, education may generate a detection bias where ceiling effects on cognitive tests prevent those with higher education from meeting diagnostic thresholds despite cognitive decline. In addition, education is a surrogate for early-life factors including nutrition, socioeconomic status and intelligence, therefore influencing risk factors in later life such as occupation, occupational exposures and health habits.

Similar to rodents, reorganization of the circuits recruited for memory processing also appears to take place in the aged human brain. This could be demonstrated by 
fMRI comparing a group composed of 20-year-old individuals to a group of 60 -year-old individuals, although these two groups had an equivalent cognitive performance [37]. During memory processing, fMRI in the aged group revealed a relative decreased activation of the medial temporal areas, whereas an increase could be observed in the ventral and prefrontal cortex, as well as in some additional frontal and parietal regions $[37,38]$. One could speculate that association with previously acquired experiences could be involved in the performance of current memory tasks in order to facilitate execution or compensate for some deficits.

More evidence that the young and aged brains react differently to the same stimulus has been seen in the analysis of antidepressant action on the neurogenesis in various groups of age. Hence, although young mice already bear high levels of neurogenesis, chronic administration of fluoxetine (an antidepressant of the SSRI family) was able to increase the generation of new neurons by as much as $75 \%$ [8]. On the other side, in aged animals and patients, chronic treatment with antidepressants did not appear to increase neurogenesis, although they are known to be clinically beneficial $[8,39]$. Hence, would an increase of neurogenesis levels in aged individuals facilitate their cognitive performance, or would the aged brain be unable to integrate this extra resource due to its extensive processing reorganization?

Interestingly, several studies substantiate the hypothesis that an 'enriched environment' is also beneficial for the cognitive performance of the elderly. Thus, the level of engagement with the environment, including cognitive, physical and social activities, has been suggested to influence cognitive health in old age. Moreover, a moderate reduction of the risks of cognitive impairments and dementia was reported in aged individuals assigned to physical or cognitive intervention programs [40,41]. Besides benefits on cardiovascular risk factors, underlying mechanisms influencing the cognitive status may include effects on neurogenesis, dendritic sprouting and electrophysiological properties.

Furthermore, participation in cognitively stimulating activities in earlier life has also been shown to be of benefit against cognitive deficits and dementia in aging. For example, more intensive leisure-time physical activity in midlife was associated with a reduced risk of dementia $[42,43]$, whereas pure occupational physical activity, which is often associated with low intellectual demand, or leisure activities with low cognitive demand, such as watching television, failed to show this protective effect or could even be detrimental $[44,45]$.
In conclusion, recent reports addressing the functional relevance of neurogenesis in the hippocampus revealed that the addition of new neurons constitutes a unique form of plasticity facilitating the performance of hippocampal-dependent tasks and the optimization of hippocampal processing circuitries. In addition, the level of neurogenesis required for the proper accomplishment of a specific cognitive task appears to be inversely proportional to previous exposure to cognitively demanding challenges. As neurogenesis levels taper off with aging, it ensues that cognitive performance becomes gradually more dependent on gathered experience. Therefore, maintaining a life-long cognitively stimulating environment and physical exercise routine may contribute to a healthy cognitive status in the elderly by assuring a continuous optimization of information processing capacity within the hippocampus and higher levels of ongoing neurogenesis. However, the possibility of reverse causality should always be borne in mind, i.e. low levels of social and cognitive activities may be an early sign of risks for cognitive decline, given that neuropathologies leading to cognitive impairments and dementia appear to begin decades before the symptoms are assigned.

\section{Acknowledgements}

We are thankful for the financial support from the State Government of Salzburg (Austria), the Bavarian State Ministry of Sciences, Research and Arts (ForNeuroCell2 grant) and the German Federal Ministry of Education and Research (BMBF grants 01GN0978 and 01GG0706).

References

1 Eriksson PS, Perfilieva E, Bjork-Eriksson T, Alborn AM, Nordborg C, Peterson DA, Gage FH: Neurogenesis in the adult human hippocampus. Nat Med 1998;4:1313-1317.

$\checkmark 2$ Brown JP, Couillard-Despres S, CooperKuhn CM, Winkler J, Aigner L, Kuhn HG: Transient expression of doublecortin during adult neurogenesis. J Comp Neurol 2003; 467:1-10.

3 Wachs FP, Couillard-Despres S, Engelhardt M, Wilhelm D, Ploetz S, Vroemen M, Kaesbauer J, Uyanik G, Klucken J, Karl C, Tebbing J, Svendsen C, Weidner N, Kuhn HG, Winkler J, Aigner L: High efficacy of clonal growth and expansion of adult neural stem cells. Lab Invest 2003;83:949-962.

-4 Schanzer A, Wachs FP, Wilhelm D, Acker T, Cooper-Kuhn C, Beck H, Winkler J, Aigner L, Plate KH, Kuhn HG: Direct stimulation of adult neural stem cells in vitro and neurogenesis in vivo by vascular endothelial growth factor. Brain Pathol 2004;14:237-248. 
5 Kempermann G, Kuhn HG, Gage FH: More hippocampal neurons in adult mice living in an enriched environment. Nature 1997;386: 493-495.

-6 van Praag H, Kempermann G, Gage FH: Running increases cell proliferation and neurogenesis in the adult mouse dentate gyrus. Nat Neurosci 1999;2:266-270.

-7 Couillard-Despres S, Winner B, Karl C, Lindemann G, Schmid P, Aigner R, Laemke J, Bogdahn U, Winkler J, Bischofberger J, Aigner L: Targeted transgene expression in neuronal precursors: watching young neurons in the old brain. Eur J Neurosci 2006;24: 1535-1545.

8 Couillard-Despres S, Wuertinger C, Kandasamy M, Caioni M, Stadler K, Aigner R, Bogdahn U, Aigner L: Ageing abolishes the effects of fluoxetine on neurogenesis. Mol Psychiatry 2009;14:856-864.

$\checkmark 9$ Tanapat P, Galea LA, Gould E: Stress inhibits the proliferation of granule cell precursors in the developing dentate gyrus. Int J Dev Neurosci 1998;16:235-239.

-10 Biebl M, Cooper CM, Winkler J, Kuhn HG: Analysis of neurogenesis and programmed cell death reveals a self-renewing capacity in the adult rat brain. Neurosci Lett 2000;291: $17-20$.

-11 Winner B, Cooper-Kuhn CM, Aigner R, Winkler J, Kuhn HG: Long-term survival and cell death of newly generated neurons in the adult rat olfactory bulb. Eur J Neurosci 2002;16:1681-1689.

12 Rapp PR, Gallagher M: Preserved neuron number in the hippocampus of aged rats with spatial learning deficits. Proc Natl Acad Sci USA 1996;93:9926-9930.

-13 Schmidt-Hieber C, Jonas P, Bischofberger J: Enhanced synaptic plasticity in newly generated granule cells of the adult hippocampus. Nature 2004;429:184-187.

-14 Lazic SE: Relating hippocampal neurogenesis to behavior: the dangers of ignoring confounding variables. Neurobiol Aging 2010; 31:2169-2171, discussion 2172-2175.

-15 Bizon JL, Lee HJ, Gallagher M: Neurogenesis in a rat model of age-related cognitive decline. Aging Cell 2004;3:227-234.

$\checkmark 16$ Deng W, Saxe MD, Gallina IS, Gage FH: Adult-born hippocampal dentate granule cells undergoing maturation modulate learning and memory in the brain. J Neurosci 2009;29:13532-13542.

$\checkmark 17$ Gould E, Beylin A, Tanapat P, Reeves A, Shors TJ: Learning enhances adult neurogenesis in the hippocampal formation. $\mathrm{Na}$ ture Neurosci 1999;2:260-265.

-18 Tronel S, Fabre A, Charrier V, Oliet SH, Gage $\mathrm{FH}$, Abrous DN: Spatial learning sculpts the dendritic arbor of adult-born hippocampal neurons. Proc Natl Acad Sci USA 2010;107: 7963-7968.

19 Ge S, Yang CH, Hsu KS, Ming GL, Song H: A critical period for enhanced synaptic plasticity in newly generated neurons of the adult brain. Neuron 2007;54:559-566.
20 Kim JS, Lee HJ, Kim JC, Kang SS, Bae CS, Shin T, Jin JK, Kim SH, Wang H, Moon C: Transient impairment of hippocampus-dependent learning and memory in relatively low-dose of acute radiation syndrome is associated with inhibition of hippocampal neurogenesis. J Radiat Res (Tokyo) 2008;49: 517-526.

21 Winocur G, Wojtowicz JM, Sekeres M, Snyder JS, Wang S: Inhibition of neurogenesis interferes with hippocampus-dependent memory function. Hippocampus 2006;16: 296-304.

22 Shors TJ, Miesegaes G, Beylin A, Zhao M, Rydel T, Gould E: Neurogenesis in the adult is involved in the formation of trace memories. Nature 2001;410:372-376.

23 Dupret D, Revest JM, Koehl M, Ichas F, De Giorgi F, Costet P, Abrous DN, Piazza PV: Spatial relational memory requires hippocampal adult neurogenesis. PLoS One 2008; 3:e1959.

24 Imayoshi I, Sakamoto M, Ohtsuka T, Takao K, Miyakawa T, Yamaguchi M, Mori K, Ikeda T, Itohara S, Kageyama R: Roles of continuous neurogenesis in the structural and functional integrity of the adult forebrain. Nat Neurosci 2008;11:1153-1161.

-25 Zhang CL, Zou Y, He W, Gage FH, Evans RM: A role for adult TLX-positive neural stem cells in learning and behaviour. Nature 2008;451:1004-1007.

26 Creer DJ, Romberg C, Saksida LM, van Praag $\mathrm{H}$, Bussey TJ: Running enhances spatial pattern separation in mice. Proc Natl Acad Sci USA;107:2367-2372.

-27 Jaholkowski P, Kiryk A, Jedynak P, Ben Abdallah NM, Knapska E, Kowalczyk A, Piechal A, Blecharz-Klin K, Figiel I, Lioudyno V, Widy-Tyszkiewicz E, Wilczynski GM, Lipp HP, Kaczmarek L, Filipkowski RK: New hippocampal neurons are not obligatory for memory formation; cyclin D2 knockout mice with no adult brain neurogenesis show learning. Learn Mem 2009;16:439-451.

28 Kowalczyk A, Filipkowski RK, Rylski M, Wilczynski GM, Konopacki FA, Jaworski J, Ciemerych MA, Sicinski P, Kaczmarek L: The critical role of cyclin D2 in adult neurogenesis. J Cell Biol 2004;167:209-213.

29 Amrein I, Dechmann DK, Winter Y, Lipp HP: Absent or low rate of adult neurogenesis in the hippocampus of bats (Chiroptera). PLoS One 2007;2:e455.

30 Bartkowska K, Djavadian RL, Taylor JR, Turlejski K: Generation recruitment and death of brain cells throughout the life cycle of Sorex shrews (Lipotyphla). Eur J Neurosci 2008;27:1710-1721.

31 Kuhn HG, Dickinson-Anson H, Gage FH: Neurogenesis in the dentate gyrus of the adult rat: age-related decrease of neuronal progenitor proliferation. J Neurosci 1996;16: 2027-2033.

32 Bizon JL, Gallagher M: Production of new cells in the rat dentate gyrus over the lifespan: relation to cognitive decline. Eur J Neurosci 2003;18:215-219.
3 Rapp PR, Stack EC, Gallagher M: Morphometric studies of the aged hippocampus: I. Volumetric analysis in behaviorally characterized rats. J Comp Neurol 1999;403:459470.

34 Kempermann G, Gage FH: Genetic determinants of adult hippocampal neurogenesis correlate with acquisition, but not probe trial performance, in the water maze task. Eur J Neurosci 2002;16:129-136.

35 Drapeau E, Mayo W, Aurousseau C, Le Moal M, Piazza PV, Abrous DN: Spatial memory performances of aged rats in the water maze predict levels of hippocampal neurogenesis. Proc Natl Acad Sci USA 2003;100:1438514390.

- 36 Tervo S, Kivipelto M, Hanninen T, Vanhanen $\mathrm{M}$, Hallikainen $\mathrm{M}$, Mannermaa A, Soininen $\mathrm{H}$ : Incidence and risk factors for mild cognitive impairment: a populationbased three-year follow-up study of cognitively healthy elderly subjects. Dement Geriatr Cogn Disord 2004;17:196-203.

37 Burgmans S, van Boxtel MP, Vuurman EF, Evers EA, Jolles J: Increased neural activation during picture encoding and retrieval in 60 -year-olds compared to 20-year-olds. Neuropsychologia 2010;48:2188-2197.

38 Grady CL: Cognitive neuroscience of aging. Ann NY Acad Sci 2008;1124:127-144.

-39 Lucassen PJ, Stumpel MW, Wang Q, Aronica E: Decreased numbers of progenitor cells but no response to antidepressant drugs in the hippocampus of elderly depressed patients. Neuropharmacology 2010;58:940-949.

40 Lautenschlager NT, Cox KL, Flicker L, Foster JK, van Bockxmeer FM, Xiao J, Greenop KR, Almeida OP: Effect of physical activity on cognitive function in older adults at risk for Alzheimer disease: a randomized trial. JAMA 2008;300:1027-1037.

-41 Levine B, Stuss DT, Winocur G, Binns MA, Fahy L, Mandic M, Bridges K, Robertson IH: Cognitive rehabilitation in the elderly: effects on strategic behavior in relation to goal management. J Int Neuropsychol Soc 2007; 13:143-152.

42 Andel R, Crowe M, Pedersen NL, Fratiglioni L, Johansson B, Gatz M: Physical exercise at midlife and risk of dementia three decades later: a population-based study of Swedish twins. J Gerontol A Biol Sci Med Sci 2008;63: 62-66.

43 Carlson MC, Helms MJ, Steffens DC, Burke JR, Potter GG, Plassman BL: Midlife activity predicts risk of dementia in older male twin pairs. Alzheimers Dement 2008;4:324-331.

44 Rovio S, Kareholt I, Viitanen M, Winblad B, Tuomilehto J, Soininen H, Nissinen A, Kivipelto M: Work-related physical activity and the risk of dementia and Alzheimer's disease. Int J Geriatr Psychiatry 2007;22:874-882.

45 Wang JY, Zhou DH, Li J, Zhang M, Deng J, Tang M, Gao C, Lian Y, Chen M: Leisure activity and risk of cognitive impairment: the Chongqing aging study. Neurology 2006;66: 911-913. 